\title{
The design of Video conferencing system based on TCP-Like protocol
}

\author{
Lei Xia \\ Department of Information Engineering, Wuhan Business University, Wuhan, China
}

\begin{abstract}
Keywords: Video conferencing system; TCP-Like protocol.
\end{abstract}
\begin{abstract}
With the rapid development of the Internet, applications such as video and audio on the internet is also rapidly developing, IP video monitor and IP video conference is now widely used in various fields. These applications play an important role in the national economy and our daily lives, Video conferencing system is a multimedia communication system based on the packet switching network. According to the characteristics of multimedia network communication with TCP-Like protocol, a system objects oriented implementation architecture is proposed and the objects in the system are described in detail. Finally, a detailed implementation process of the terminal was designed.
\end{abstract}

\section{Introduction}

With the development of computer communication technologies as well as the increasing of Internet bandwidth, videophone and video conferencing system based on packet switching network have been widely used in various fields.In order to make different products manufacturers circulating, the international telecommunications union (ITU) created video conference communication standards to run on packet switching network.

Real-time multimedia streaming will be the main transmission objective on the next generation network, which could have large effect on our lives. There exist many advantages such as reliable transmission, rapid reaction to network congestion, TCP friendliness, acceptableness to fire walls, convenient for implementation and maintenance and so on while using TCP to transmitting real-time multimedia streaming. However, on the other hand, the disadvantages are obvious as follows. The sending rate is unable to be adaptive to the available network bandwidth. The re-transmission arisen from TCP would likely increase the delay fitter and confusion of multimedia packet. These disadvantages decrease notablely the quality of service of real-time multimedia streaming. Armed at the above problems, performance analysis of real-time multimedia streaming transmission, sending-delays prediction model, rate adaptive transmitting scheme and its application are researched in this thesis and the main creative achievements included are the following five aspects.In this paper, video conference system has been successfully applied in the actual network data processing based on the TCP algorithms, it proved that this technology is a feasible audio and video flows capturing technology

\section{Average Delay Jitter Model}

The end-to-end delays of real-time video are dissected by deriving and simulating, and it is discoveried that the waiting-delays are the main part of end-to-end delays of real-time video and the sending-delays are the critical factor impacting on the end-to-end delays. Two ways to decrease the end-to-end delays and jitters are presented, which are reducing the waiting-delays and cutting down the sending-delays. Since the sending-delays are straightly and rigidly managed by the TCP stack, it is impossible for applications to control it. Therefore, a method to decrease the end-to-end delays by predicting and judging the sending-delays is proposed as follows. Predict the sending-delays of a video frame and if it can not achieve the requirement mentioned above, then the video frame will be selectively discarded. Utilizing the method of predicting and judging, the sending-delays of all the video frames deliveried are little. The simulation results show that the end-to-end delays of real-time video can be cut down notablely by using the method. 
The delay jitter of TCP-like algorithm is set as the variation of RTP data frame arrival time. Set Di as the arrival time of $\mathrm{i}$ frame and the jitter is expressed by formula (1):

Jitter $=\left|D_{i}-D_{i-1}\right|$

During the transmission of TCP-like algorithm, define two adjacent times between package loss with the sign of $r \neq b$, as TD, in which, $r$ is the total number of actually received and lost frames, $b$ is the feedback rate. The window variation of TD period is similar with standard TCP model period of receiving repeated acknowledgment frame. Assuming that $\mathrm{Y}$ is correctly received frames during TD period, we can obtain the expected value of Y according to standard TCP model, shown in formula (2):

$$
E(Y)=\frac{1-p}{p}+\sqrt{\frac{8}{3 b p}}
$$

The expected value of $\mathrm{W}$, which is the last window during TD period, is shown in formula (3):

$$
E(W)=\sqrt{\frac{8}{3 b p}}
$$

It can be conclude that the delay of each frame is RTT/2 and the jitter delay is 0 . When the TD period ends, the average delay of received frame will increase if has frame loss, what is similar with standard TCP receiving repeated acknowledgment? Through standard TCP model analysis we can know that the average delay jitter is RTT/ (2b) at this time. Therefore, the delay jitter of first frame at the beginning of TD can be set as RTT/ (2b) in the time of starting TD period.

The expected value of average jitter can be obtained based on above model analysis, assuming that the transmitted lost frame is up to E (W) actually in Tout period, shown as formula (4).

$$
E(\text { Jitter })=\frac{\frac{1}{E(n)}|2 T 0-R T T|+\left(1-\frac{1}{E(n)}\right)\left(\frac{R T T}{b}\right)}{E(Y)-\frac{1}{E(n)} E(W)}
$$

In the formula, $1 / E(n)$ can be understood as the timeout probability of TD period, set $Q=1 / E(n)$ and (4) can be expressed as (5):

$$
E(\text { Jitter })=\frac{Q(2 T O-R T T)+(1-Q)\left(\frac{R T T}{b}\right)}{E(Y)-Q \cdot E(W)}
$$

It will be obtained from standard TCP model that, when $\mathrm{p}->0$,

$$
E(Q)=\frac{1}{E(W)}=\sqrt{\frac{3 b p}{8}}
$$

Substitute formula (2) (3) (6) into (5), the estimated value of jitter can be obtained as formula (7) after finishing:

$$
E(\text { Jitter })=\frac{p\left(2 T 0 \sqrt{\frac{3 b p}{8}}+\frac{R T T}{b}-\frac{(1+b) R T T}{b} \sqrt{\frac{3 b p}{8}}\right)}{1-2 p+\sqrt{\frac{8 p}{3 b}}}
$$

When p is small, the denominator of formula (1) is about 1 , and then the estimated value can be expressed as formula (8):

$$
E(\text { Jitter })=p\left(2 T 0 \sqrt{\frac{3 b p}{8}}+\frac{R T T}{b}-\frac{(1+b) R T T}{b} \sqrt{\frac{3 b p}{8}}\right)
$$

\section{Principle of TCP-like Algorithm}

TCP-like algorithm is composed of receiving acknowledgment module, window control module, transmission control module and clock control module, etc. The size of window is adjusted by the 
window control module according to acknowledgment frame received by receiving module and timeout situation which is obtained from clock control module. The transmission control module transmits streaming media data according to the size of window, the clock control module calculates and confirms timeout clock value and handles information of other modules and the receiving acknowledgment module transmits acknowledgment frame to the sender according to received data frame. The algorithm structure is shown in Fig.1:

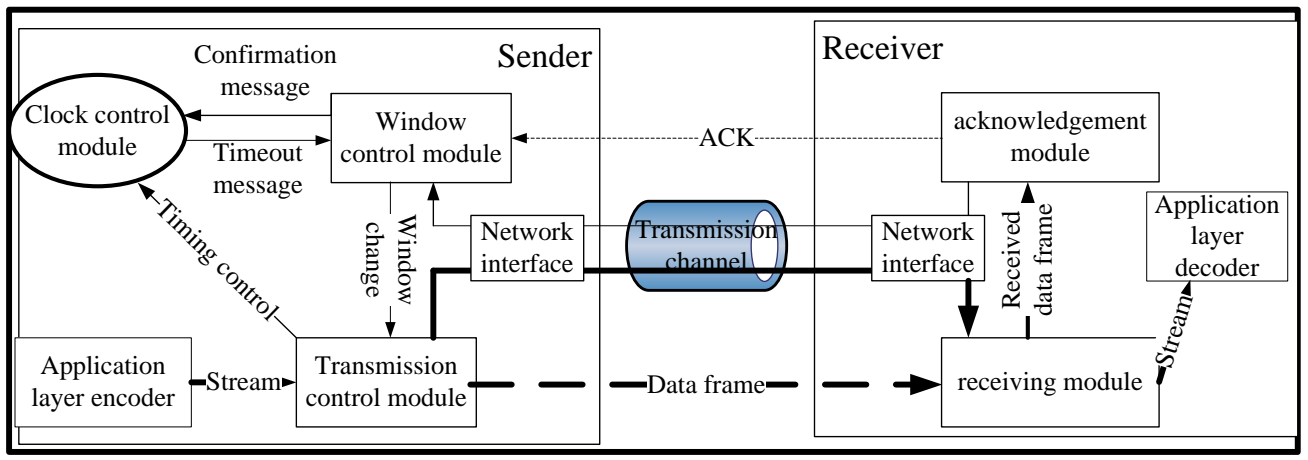

Fig.1 TCP-like Algorithm Structure Model

TCP-like algorithm uses TCP acknowledgment mechanism. RTCP receiving report (SR, RR frame) will be transmitted to sender to confirm the received last data frame after the receiving terminal receives RTP data frame required by feedback rate or the clock timeout. The transmission rate of RTP data frame is adjusted by using improved AIMD algorithm control window. In order to achieve more stable rate variation and realize a stronger self-synchronization between flow and control in the stream media transmission based on RTP/RTCP, conversion relationship and control parameters of the original TCP window control mechanism (slow start, congestion avoidance and fast recovery) is improved. At the same time, the re-transmission mechanism of TCP is completely abandoned to prevent further congestion and long-term transmission pause caused by waiting.

\section{Video conference system structure}

The transmission information contained in the video conference system are audio, video, data and control information.All of the information flow using h.225 protocol for packaging and delivery.In order to achieve the transmission the information flow in the conference system, the multimedia video conference system is mainly composed of terminals, network Gateway (Gatekeeper), Gateway (Gateway), multi-point controller (MC),multi-point processor (MP) and multi-point control unit (MCU) and so on, as shown in figure 2.

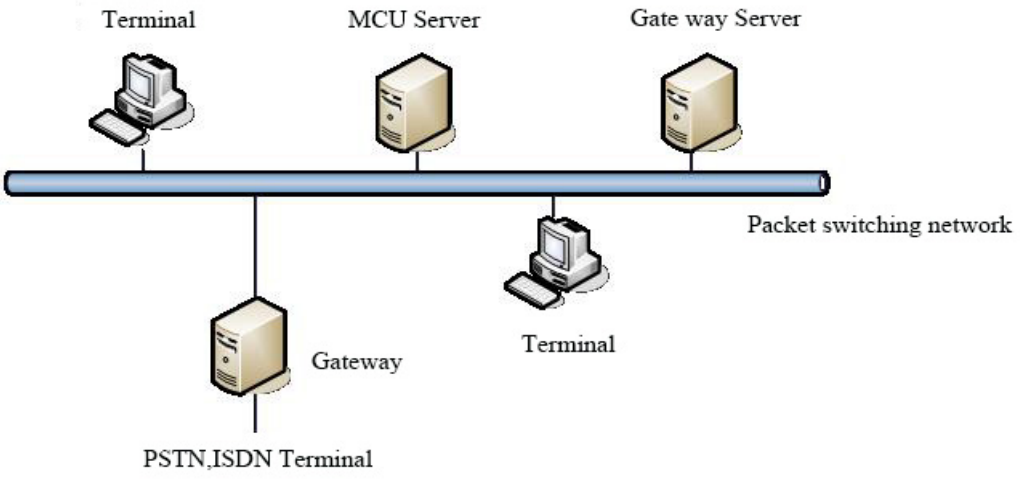

Fig 2 The multimedia video conference system

\section{Conclusion}


Video conference system is a multimedia communication system based on packet switching network including all calling signal, control signal, multimedia data finally is transmitting in the form of IP packet.The process of transmitting real-time multimedia via TCP is deeply analyzed and then the requirement of transmitting real-time audio with acceptable playing performance via TCP is pointed as that the TCP MSS should be equal with the size of a audio frame. However, the transmitting efficiency of TCP will be very low under this condition so that it is unfeasible in the Internet. The requirement of transmitting real-time video is also pointed as that the video frame sending-delays should be less than the results that play out buffer delays subtract half of the round trip time (RTT).The simulation results show that the requirement presented is reasonable.The system component solves the following questions:1.Multi-channel data can be recorded in the same file according to a certain criterion.2.Just only using several interface to complete record and play, regardless of the internal operation of the file.3. Location file position efficiently base on the seeking time.

\section{Reference}

[1] Xie Yingling. Video conference media streaming capture technology [J]. Journal of tele-com technology research. 2012, 42 (4) : 12-16.

[2] Li jie, zheng-mao wu. Mobile terminal to realize the video conference solution research [J]. Journal of TV technology. 2012, 36 (19) : 193-195.

[3] Zhizhong Zhao. Video gateway based on Asterisk study [J]. Information systems and networks. 2013, 43 (6) : 6-9.

[4] ITU-T Recommendation. H.323 Packed-based multimedia communications systems[S]. 2003, 07. 